\title{
TITLE:
}

\section{$<$ Note $>$ Wild Chimpanzees at Mahale are not Manually Lateralised for Throwing}

\section{$\operatorname{AUTHOR}(S)$ :}

Nishida, Toshisada; McGrew, William C.; Marchant, Linda F.

\section{CITATION:}

Nishida, Toshisada ... [et al]. < Note> Wild Chimpanzees at Mahale are not Manually Lateralised for Throwing. Pan Africa News 2012, 19(2): 2123

ISSUE DATE:

2012-12

URL:

http://hdl.handle.net/2433/168178

RIGHT:

Copyright (C) Pan Africa News. 
339-356.

3. Williams JM, Lonsdorf EV, Wilson ML, SchumacherStankey J, Goodall J, Pusey AE, 2008. Cause of death in the Kasekela chimpanzees of Gombe National Park, Tanzania. Am J Primatol 70:766-777.

4. Sugiyama Y and Fujita S 2010. The demography and reproductive parameters of Bossou chimpanzees. In: The Chimpanzees of Bossou and Nimba. Matsuzawa T, Humle T, Sugiyama Y (eds), Springer, Tokyo, pp. 23-34.

5. Boesch C, Boesch-Achermann H 2000. The Chimpanzees of the Taï Forest. Oxford University Press, Oxford.

6. Cutler RG 1984. Carotenoids and retinol: Their possible importance in determining longevity of primate species. Proc Natl Acad Sci USA 81:7627-7631.

7. Goodall J 1986. The Chimpanzees of Gombe. Harvard University Press, Cambridge, MA.

8. Emery Thompson M, Jones JH, Pusey AE, BrewerMarsden S, Goodall J, Marsden D, Matsuzawa T, Nishida T, Reynolds V, Sugiyama Y, Wrangham RW 2007. Aging and fertility patterns in wild chimpanzees provide insights into the evolution of menopause. Curr Biol 17:2150-2156.

9. Wrangham RW, Clark AP, Isabirye-Basuta G, 1992. Female social relationships and social organization of Kibale Forest chimpanzees. In: Topics in Primatology, Vol.1. Nishida T, McGrew WC, Marler P, Pickford M, de Waal FBM (eds), University of Tokyo Press, Tokyo, pp. $81-98$.

10.Hill K, Boesch C, Goodall J, Pusey A, Williams J, Wrangham R 2001. Mortality rates among wild chimpanzees. J Hum Evol 40:437-450.

\section{<NOTE>}

Wild Chimpanzees at Mahale are not Manually Lateralised for Throwing

\author{
Toshisada Nishida1, William C. \\ McGrew ${ }^{2} \&$ Linda F. Marchant ${ }^{3}$ \\ 1 Deceased \\ 2 Div. of Biological Anthropology, Dept. of Archaeology \& \\ Anthropology, Univ. of Cambridge, UK \\ 3 Dept. of Anthropology, Miami University (Ohio), USA \\ (E-mail:wcm21@cam.ac.uk)
}

\section{INTRODUCTION}

The importance of throwing has figured consistently and prominently in scenarios about the evolutionary origins of human behaviour ${ }^{1,2}$ The utility of imparting force to airborne projectile weapons, launched ballistically, is obvious, whether to deter, punish or subdue predators, prey, or competitors. Other functions of throwing are less obvious but could be equally useful, such as bringing down hanging fruit, gaining others' attention, shattering large objects into fragments, etc. Scenarios have stressed a number of important variables, such as posture (especially bipedality), sensorimotor skill (especially hand-eye coordination), cerebral asymmetry, etc. Calvin ${ }^{1}$ linked all of these to the evolutionary origins of language. However, few of these ideas have been tested empirically.

One way to tackle evolutionary aspects of human throwing is to look at throwing performance in our nearest living relations. Although throwing has been known in both wild ${ }^{3}$ and captive ${ }^{4}$ chimpanzees for almost a century, quantitative studies are few; often they are sub- sumed in broader studies of manual behaviour, e.g. ref. 5. Furthermore, studies often do not distinguish between aimed (targeted) throwing versus unaimed (perhaps better termed hurling) throwing, underarm versus overarm delivery, or one-handed versus two-handed throwing.

Several studies of manual laterality in throwing by captive chimpanzees have been published, and all have reported population-level, right-sided bias ${ }^{6-8}$. Others have incorporated these reported findings into comprehensive accounts of the origin of handedness, e.g. ref. 9

We can find no published quantitative data on manual laterality in throwing from any non-human primate species in nature, much less from chimpanzees. One obvious reason for this absence is that, unlike in the artificial conditions of captivity, wild primates throw only rarely. Here we report such a dataset, collected ethologically from a population of chimpanzees in nature, over an extended period, in an effort to balance the picture.

\section{METHODS}

The subjects were the eastern chimpanzees (Pan troglodytes schweinfurthii) of $\mathrm{M}$ group in the Mahale Mountains of western Tanzania ${ }^{10,11}$. This population has been studied since 1965, making it the second-oldest field study of chimpanzees; the apes are fully habituated to close-range behavioural observation. The Mahale ethogram is well-known and described; Nishida et al..$^{12}$ listed 10 kinds of throwing.

We recorded all observed occurrences of throwing, using ad lib. sampling, that is, regardless of whether behaviour was being scan-sampled or focal-subjectsampled $^{13}$. We interrupted normal protocols, in order to note the identity of the thrower, target, etc. Nishida recorded his data from 1999-2004, when he mostly followed males, hence the shortage of data on females. McGrew and Marchant recorded their data in 1996. Here we report data only on unimanual versus bimanual data, and on the hand(s) involved. Each throw is considered an independent data-point, as the chimpanzees always repositioned themselves between throws, usually to pick up another object.

\section{RESULTS}

We recorded 556 throws by 16 individuals (Table 1); the median number of throws per subject was 33 (range: 9-72). Of these, 63 (11\%) were done two-handed, usually in agonistic charging displays; almost half of these were done by two high-ranking adult males (DE, $n=17$; FN, $n$ $=13$ ). These appeared to be unaimed and perforce were done bipedally; they are considered no further here.

One-handed throwing was unlateralized. Only 4 (BB, $\mathrm{CT}, \mathrm{IV}, \mathrm{OR})$ of the 16 individuals showed statistically significant (Binomial test, two-tailed, $p<0.05$ ) laterality; three were biased to the right versus one to the left The remaining 12 subjects showed ambilateral performance. Overall, nine individuals showed (non-significantly) more right-sided throws, six showed left-sided throws (a nonsignificant difference, $n=15, x=6, p=0.60$ ), and one was tied. Descriptively overall, of one-handed throws, 226 (46\%) were done with the left hand versus 267 (54\%) with the right hand. 
Table 1. Laterality of throwing by wild chimpanzees ( $n=16)$ of Mahale's M group.

\begin{tabular}{|c|c|c|c|c|c|c|}
\hline Name & $\begin{array}{c}\text { Year of } \\
\text { Birth }\end{array}$ & $\begin{array}{c}\text { Total } \\
\text { Throws }\end{array}$ & Both Hands & Left Hand & Right Hand & $\begin{array}{l}\text { R vs. L } \\
\text { p }<0.05\end{array}$ \\
\hline$\overline{\mathrm{AL}}$ & 1982 & 46 & 1 & 21 & 24 & \\
\hline $\mathrm{BB}$ & 1981 & 37 & 2 & 26 & 9 & $\mathrm{~L}$ \\
\hline $\mathrm{CD}$ & 1991 & 34 & 1 & 18 & 15 & \\
\hline $\mathrm{CE}$ & 1998 & 11 & 0 & 3 & 8 & \\
\hline CT & 1985 & 32 & 1 & 6 & 25 & $\mathrm{R}$ \\
\hline $\mathrm{DE}$ & 1963 & 27 & 17 & 3 & 7 & \\
\hline DG & 1981 & 21 & 3 & 10 & 8 & \\
\hline DW & 1988 & 36 & 2 & 17 & 17 & \\
\hline $\mathrm{FN}$ & 1978 & 70 & 13 & 27 & 30 & \\
\hline IV & 1993 & 20 & 1 & 4 & 15 & $\mathrm{R}$ \\
\hline $\mathrm{MC}$ & 1996 & 64 & 9 & 28 & 27 & \\
\hline OR & 1991 & 47 & 7 & 10 & 30 & $\mathrm{R}$ \\
\hline OS & 1998 & 14 & 0 & 5 & 9 & \\
\hline PM & 1988 & 16 & 1 & 10 & 5 & \\
\hline PR & 1991 & 72 & 4 & 35 & 33 & \\
\hline XM & 1995 & 9 & 1 & 3 & 5 & \\
\hline TOTAL & & 556 & 63 & $226(46 \%)$ & $267(54 \%)$ & \\
\hline
\end{tabular}

\section{DISCUSSION}

These results clearly contrast with those from the most thorough study done in captivity: Hopkins et al. ${ }^{7}$ found that of 89 subjects with six or more data-points, 50 were right-biased, 23 were left-biased, and 16 were unbiased. We have no explanation of this remarkable difference, although it joins a long list of behavioural contrasts between nature and captivity, in which captive chimpanzees are lateralised, while wild chimpanzees are not (see summary in ref. 14). We can find no reports of twohanded throwing by captive chimpanzees; whether this is because such behaviour was not reported, or because it is absent, remains to be seen.

Earlier, McGrew and Marchant ${ }^{15}$ hypothesised that captive chimpanzees might be biased toward rightsidedness by their lengthy (sometimes lifetime) association with human caretakers, most of whom will have been right-handed. However, a recent extensive study of four great ape species (see below) found species-level rightsided laterality in captive chimpanzees, at least based on a single bimanual measure ${ }^{16}$.

The implications for the evolutionary origins of human handedness are no clearer than the present state of play of contradictory studies of behavioural laterality. Handedness in living Homo sapiens could be a derived trait (of uncertain time-depth) or a primitive trait that could date back as far as the last common ancestor of the present African apes and humans (perhaps as long ago as 6-7 million years). Results on manual laterality for the only living Asian great ape, the orang-utan, Pongo pygmaeus, are confusing, as according to Hopkins et al. ${ }^{16}$, they are a left-handed species.

What is clear is that chimpanzees both in captivity and in nature habitually throw, despite being non-linguistic quadrupeds. Thus it is not necessary to invoke bipedal stance or co-evolved language to explain the evolutionary origins of throwing in the hominin lineage, and if the results from nature are to be believed, neither is it necessary to link throwing to manual lateralisation.

\section{ACKNOWLEDGEMENTS}

We thank: the Tanzania Commission for Science and Technology (COSTECH/UTAFITI), Serengeti (now Tanzania) Wildlife Research Institute (SWRI/TAWIRI), Tanzania National Parks (TANAPA) for authorisation to do the research; H.M. Nguli, E.T. Massawe, B.C. Mwasaga, and J.V. Wakibara for special administrative assistance; M. Huffman, K. Kawanaka, M. Nakamura, and S. Uehara for field collaboration; Philip and Elaina Hampton Fund (Miami University) for financial assistance to LFM \& WCM, and Japanese MEXT \#12375003, \#16255007 for financial assistance to TN. Toshisada Nishida died on 7 June, 2011 and is much missed.

\section{REFERENCES}

1. Calvin WH 1983. The Throwing Madonna: Essays on the Brain. McGraw-Hill, New York.

2. Isaac B 1987. Throwing and human evolution. Afr Archaeol $\operatorname{Rev}$ 5:3-17.

3. Goodall J 1964. Tool-using and aimed throwing in a community of free-living chimpanzees. Nature 201:1264-1266.

4. Köhler W 1927. The Mentality of Apes. 2nd edit. Kegan Paul, Trench, Trubner, London.

5. Marchant LF 1983. Hand Preferences among Captive Island Groups of Chimpanzees. PhD dissertation, Rutgers, The State University of New Jersey, New Brunswick, NJ.

6. Hopkins WD, Bard KA, Jones A, Bales SA 1993. Chimpanzee hand preference in throwing and infant cradling: Implications for the origin of human handedness. Curr Anthropol 34:786-790.

7. Hopkins WD, Russell JL, Cantalupo C, Freeman H, Schapiro SJ 2005. Factors influencing the prevalence and handedness for throwing in captive chimpanzees (Pan troglodytes). J Comp Psychol 119:363-370.

8. Hopkins WD, Russell JL, Schaeffer JA 2012. The neural and cognitive correlates of aimed throwing in chimpanzees: A magnetic resonance image and behavioural study 
on a unique form of social tool use. Phil Trans Roy Soc B 367:37-47.

9. Forrester GS, Quaresmini C, Leavens DA, Mareschal D, Thomas MSC 2013. Human handedness: An inherited evolutionary trait. Behav Brain Res 237:200-206.

10.Nishida T (ed) 1990. The Chimpanzees of the Mahale Mountains: Sexual and Life History Strategies. University of Tokyo Press, Tokyo.

11. Nishida T 2012. Chimpanzees of the Lakeshore. Natural History and Culture at Mahale. Cambridge University Press, Cambridge.

12.Nishida T, Zamma K, Matsusaka T, Inaba A, McGrew WC 2010. Chimpanzee Behavior in the Wild: An Audio-visual Encyclopedia. Springer, Tokyo.

13. Martin P, Bateson P 2007. Measuring Behaviour: An Introductory Guide. 3rd edit. Cambridge University Press, Cambridge.

14. McGrew WC, Marchant LF 1997. On the other hand: Current issues in and meta-analysis of the behavioral laterality of hand function in nonhuman primates. Yearb Phys Anthropol 40:201-232.

15. McGrew WC, Marchant LF 2001. Ethological study of manual laterality in the chimpanzees of the Mahale Mountains, Tanzania. Behaviour 138:329-358.

16. Hopkins WD, Phillips KA, Bania A, Calcutt SE, Gardner M, Russell J, Schaeffer J, Lonsdorf EV, Ross SR, Schapiro SJ 2011. Hand preferences for coordinated bimanual actions in 777 great apes: Implications for the evolution of handedness in Hominins. J Hum Evol 60:605-611.

\section{<NOTE>}

Playful Drumming by Immature Wild Chimpanzees at Mahale: Do They Enjoy Making Sounds?

\author{
Takahisa Matsusaka \\ Kansai University, Japan \\ (E-mail: matsusaka.t@gmail.com)
}

\section{INTRODUCTION}

Before the Mahale Mountains National Park was established in 1985, several villages of Tongwe people lived in the forest. Although many years have passed since the people left, we can still sometimes find the remains of human artifacts on the forest floor while observing chimpanzees. Here, I report the rare cases where immature chimpanzees found and drummed on clay pots discarded by the former villagers and discuss their playful nature in relation to the origin of music.

\section{OBSERVATIONS}

(1) Case of an 11-Year-Old Adolescent Male, Cadmus (CD) (25 Nov. 2002)

While CD was playing with a 4-year-old male infant (OS) in a thicket, CD found at 10:13:59 a clay cooking pot (about $40-\mathrm{cm}$ diameter, $30-\mathrm{cm}$ high, with a $14-\mathrm{cm}$ diameter hole at the bottom). He lifted it with his left hand and approached OS with a play-face. OS approached and touched $\mathrm{CD}$, but soon returned to his mother to suckle.

At 10:15:29, an estrous adolescent female (QA) showed leaf-clipping courtship near $\mathrm{CD}$, who did not respond. At 10:15:39, CD lifted the pot with both hands, put it on his head like a helmet, and tapped 31 times on its side with his right palm. Then he stamped on the ground four times and pounded the pot three times with his left fist. After stamping on the ground twice, he again tapped on the pot's side with his right palm 14 times (Figure 1).

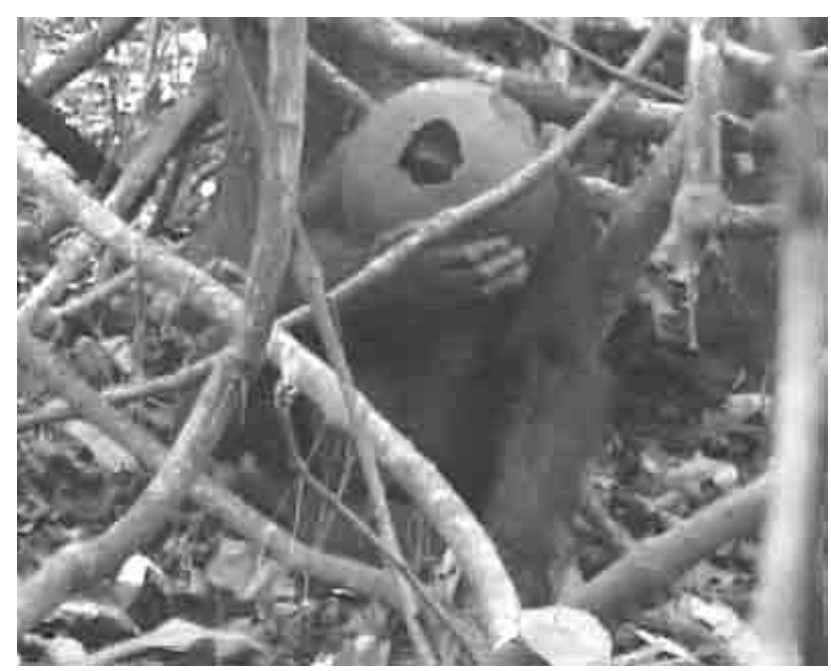

Figure 1. CD puts a pot on his head and taps it with his palm.

At 10:16:42, CD lowered his head, placed the pot on the ground, and kicked its rim twice with his right heel. Then he lowered one side of the rim with his right hand and kicked the rim with his right heel ten times. At 10:17:19, CD stood up, kicked backward, and pushed the pot with his right sole. The pot rolled off, and he left.

In total, CD drummed 60 times: 45 times with his right palm, three times with his left fist, and 12 times with his right heel. He calmly drummed with no pilo-erection. His face was not visible when he put the pot on his head, but he drummed on it on the ground with no facial expression. He generally tapped lightly and did not make loud sounds. Although he might have heard the pot's large resonance from inside it, the sounds did not seem to attract others. No one approached CD during his drumming, even though six chimpanzees rested within $10 \mathrm{~m}$ : OS and his mother, a 1-year-old male infant (TD) and his mother, an adult male (DG), and an adolescent female (QA).

\section{(2) Case of a 6-Year-Old Juvenile Male, Michio (MC) (14 Feb. 2003)}

At 10:46:56, while MC was walking in a thicket, he found a large clay water pot that was about $50-\mathrm{cm}$ high, with a $45-\mathrm{cm}$ diameter, a narrow neck, and an $18-\mathrm{cm}$ diameter mouth. He slapped the bottom of it three times with his left palm, rolled it, and slapped it once with his right palm. Then he stepped onto it with both feet, soon got off it, lowered the pot's mouth to the ground, and slapped its bottom with his left palm. He then pushed and rolled it, slapped it simultaneously with both palms, and slapped it nine times with both hands in turns. At 10:47:19, he stopped but soon started playing again in various ways (Figure 2, Video 1: available online at mahale.main.jp/PAN/19_2/19(2)_05.html): slapping the pot with his palms, pushing and rolling it, stamping on it with his soles, kicking it with his heels, stepping up onto 